\title{
Extrapulmonary tuberculosis presenting as a dumbbell tumour of the chest wall
}

\author{
Kariyawasam AGTA ${ }^{1}$, Fonseka $\mathrm{CL}^{2}$, Rasnayake $\mathrm{D}^{3}$, Singhapura $\mathrm{SDAL}^{1}$, Sanjeewa $\mathrm{ADS}^{4}$, Hewavithana $\mathrm{J}^{1}$, \\ Masakorala ND ${ }^{1}$, Dahanayake $\mathrm{NJ}^{2}$, Bodinayake $\mathrm{CK}^{2}$ \\ ${ }^{\prime}$ University Medical Unit, Teaching Hospital Karapitiya, Galle, Sri Lanka. \\ ${ }^{2}$ Department of Medicine, Faculty of Medicine, University of Ruhuna, Galle, Sri Lanka. \\ ${ }^{3}$ Chest Hospital, Welisara, Sri Lanka. \\ ${ }^{4}$ Department of Radiology, Teaching Hospital Karapitiya, Galle, Sri Lanka.
}

Correspondence: $\quad$ Dr.A.G.T.A. Kariyawasam

e-mail: thiliniaro88@gmail.com

(iD) https://orcid.org/0000-0003-4297-149X

\section{Introduction}

Tuberculosis gives rise to a significant impact on the healthcare burden in tropical countries where it can present in a variety of ways. The commonest site of tuberculous infection is the lung, while extra-pulmonary tuberculosis (TB) is observed only among $15 \%$ to $20 \%$ of all cases mostly among immune-suppressed patients (1). Musculoskeletal system is one of the well-known sites for extrapulmonary TB which accounts for nearly $1 \%$ to $3 \%$ of all TB cases (2-4). Chest wall tuberculosis is a rare entity of musculoskeletal TB constituting only $1 \%$ to $5 \%$ of musculoskeletal TB $(3,4)$. These sort of uncommon presentations are even rare among young immune competent adults. Being such rare we seldom suspect tuberculosis in young adults with no predisposition and presenting with minimal symptoms. Therefore, this case opens up new stream of thinking which is crucial in early recognition and early commencement of specific treatment for a potentially fatal disease.

\section{Case report}

A 30-year old otherwise healthy female presented with a painful swelling on her back of the chest which she had noticed about three months prior to the presentation. She noticed that it is increasing in size but denied any fever or constitutional symptoms, cough or weight loss. She was apparently well with normal appetite and had no past history or exposure to TB.
The clinical examination was unremarkable except for a fluctuant cystic lump over the posterior chest wall. It was mildly tender and had a maximum dimension of $10 \mathrm{~cm}$ and the overlying skin appeared normal. Her basic blood tests showed normal hematological and biochemical profile except for elevated ESR of $78 \mathrm{~mm} / 1^{\text {st }}$ hour with a CRP of $69 \mathrm{u} / \mathrm{L}$. The patient did not have diabetes. The chest radiograph was normal and her sputum samples were negative for acid fast bacilli and the Mantoux test was positive with a reading of $15 \mathrm{~mm}$. Ultrasound scan of the lump revealed a cystic intramuscular mass in the back of the right side of the chest wall with extension to the right perinephric space (Figure 1). The subsequent CT scan of the chest and abdomen revealed a large multilobulated intramuscular abscess in the back of the right lower chest and upper abdomen (Figure 2) mainly involving the latissimus dorsi muscle measuring $12 \mathrm{~cm}$ craniocaudally and $5 \mathrm{~cm}$ transversely. The CT scan further described the deep extensions of the abscess to the $T_{10}-T_{11}$ intercostal space and it extended to the pleural space causing a small empyema. It is further extended through the $T_{11}-T_{12}$ intercostal space into the peritoneum to result in a small pus collection posterior to the Gerota fascia of the right kidney without any involvement of the perinephric fat (Figure 3).

With the positive Mantoux and CT evidence of a cold abscess she was started on anti TB drugs without much improvement. Subsequently we decided on surgical excision of the cold abscess. 
The histology of the abscess wall revealed granulomatous inflammation with caseous necrosis, further confirming diagnosis of tuberculosis. The anti TB drugs were continued for six months under the care of respiratory team and she showed complete recovery. The wound healed without any complication such as sinus tract formation. She was well and was back to her usual health when we reviewed her six months after completing anti TB drugs.

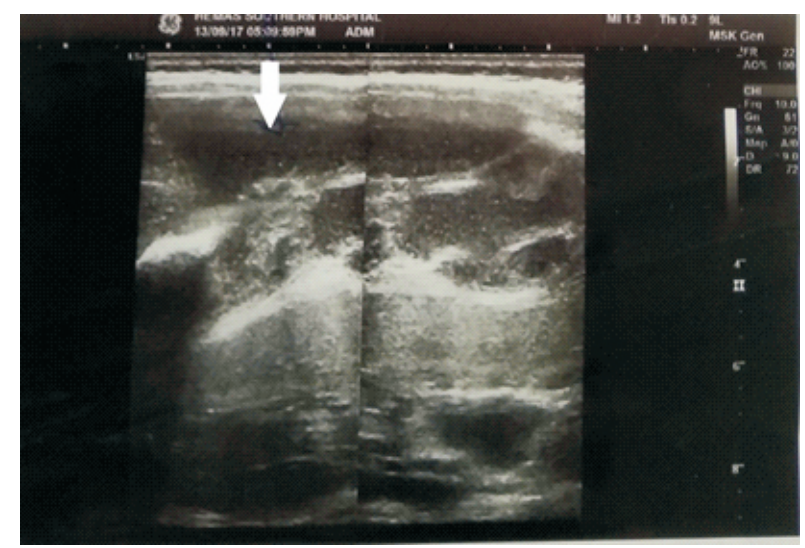

Figure 1: Ultrasound scan showing perinephric extension of the abscess

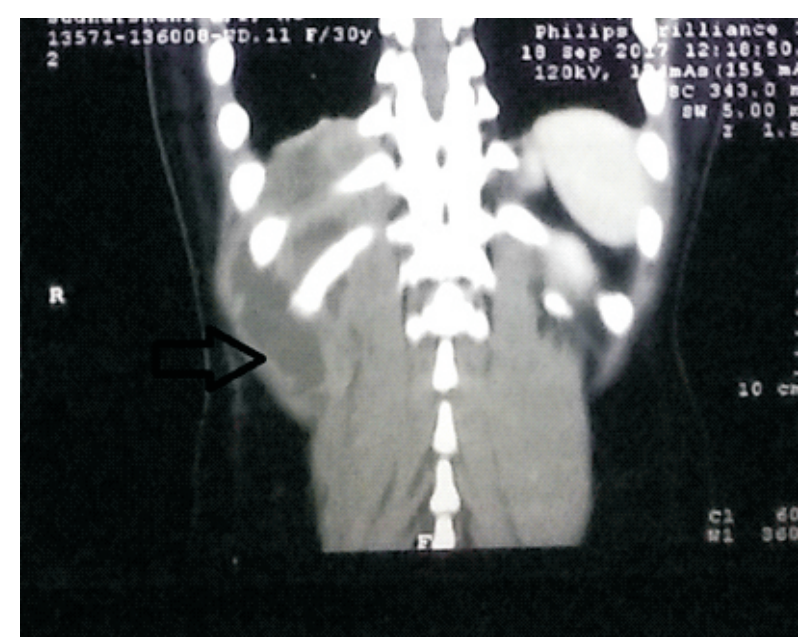

Figure 2: Coronal view of the CT showing intramuscular mass in the back of the right side of the chest wall with extension to the right perinephric space.

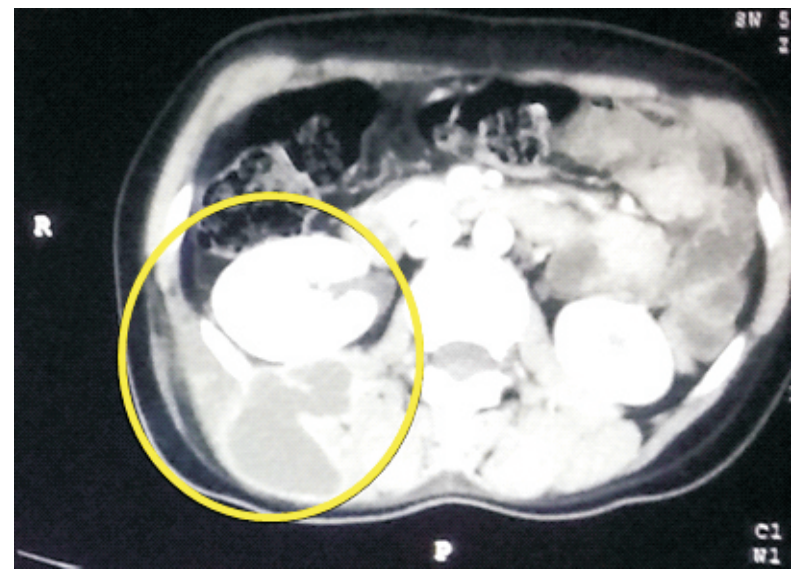

Figure 3: $\mathrm{CT}$ abdomen showing the intramuscular abscess extending into the peritoneum and posterior to the Gerota fascia of the right kidney

\section{Discussion}

It is well-known that chest wall $\mathrm{TB}$ can present as a "cold abscess." A cold abscess is described in literature as a swelling without much inflammation (5). These abscesses are made up of caseous material (6) that results from necrosis of tissue due to chronic inflammation caused by tuberculous bacilli.

Skeletal muscles are not known to get involved even in cases of disseminated tuberculosis (7) as skeletal muscle is considered to be an unfavorable site for the survival of mycobacteria reasoning out why this type of TB is rare $(7,8,9)$. According to literature though rare, the skeletal muscles which were involved in the course of TB were the pelvic muscles, anterior abdominal wall muscles and paraspinal muscles where the infection was due to secondary spread from bone or joint infection $(7,9,10)$. In such a background, the primary infection of the Latissimus dorsi muscle is an unusual form of tuberculosis. The extension of the abscess to both the retroperitoneal as well as the pleural spaces gave rise to the 'dumbbell' shape to it making this presentation further interesting. Such extension of intramuscular cold abscess of the Latissimus dorsi has not been reported earlier.

Several mechanisms are described in the literature to explain the possible ways of tuberculous bacilli gaining access to the chest wall. In an institutional study by Faure et al., described eighteen patients with chest wall cold abscess (CWCA) in which $83 \%$ had a history of tuberculosis(1), while Kyu 
Do Cho, et al., reported 12 out of 16 patients (11). This observation strongly supports a theory of activation of a dormant tuberculous focus leading to haematogenous dissemination $(3-5,12)$ as a possible mechanism. In the same study active pulmonary TB was seen in $33 \%$ of the patients (1). Another possible mechanism is that the direct extension from lymphatics of the chest wall $(1,5,12)$. This mechanism is supported by the finding of continuity of chest wall collections into enlarged caseous intrathoracic lymph nodes $(1,6)$ in about $50 \%$ of patients with CWCA. They were mainly the internal mammary nodes that were involved. Cold abscesses of chest wall are generally solitary though multiple lesions are not surprising $(5,11)$. Most of the time they resemble either a pyogenic abscess or a chest wall tumor. This close resemblance, make CWCA difficult to diagnose. In such a setting $\mathrm{CT}$ is thought to be ideal for evaluating tuberculous chest wall lesions (6). With a CT the nature and the extent of soft tissue involvement can be studied. Accompanying intrathoracic adenopathy and bone erosion are also well documented in CT (6). Histological examination is important in establishing a diagnosis of tuberculosis as well in excluding other diagnoses. This might help the diagnosis of tuberculosis by showing caseating granulomas with giant cells or revealing acid-fast bacilli in a direct smear or growing Mycobacterium tuberculosis in a culture. Needle aspiration might not be much reliable as the aspirated material may not contain typical giant cells or acid- fast bacilli. And this procedure has a low diagnostic yield $(1,5)$. Therefore, when there is a strong clinical suspicion surgical biopsy becomes the gold standard (5).

There is no consensus regarding optimal therapeutic management for CWCA. Some recommend standard anti-tuberculous drugs alone $(13,14)$ and some recommend surgical procedures while best results were reported when both were combined since it is proven to reduce the recurrences $(1,4,13$, 14). Six-month chemotherapy regimen is supposed to be adequate with successful surgical excision. In the case of non-operated patients, 9 to 12 month duration regimens are commonly used $(2,14)$. The surgical method of choice is supposed to be a radical or wide excision though it lacks adequate supportive data $(1,4,11)$. Even though only a few patients respond to anti-tuberculous medication, medical treatment should be tried first before attempting a surgical approach. After 1 - 3 months of medical treatment, surgical resection should be considered if the lesion fails to improve or gets worsened. Though TB is common in our part of the world, the management of this type of rare forms of the disease is totally based on available literature evidence which is also limited to few case reports or series. Therefore we consider reporting this kind of successfully treated cases is of utmost importance. Further evidence is necessary to shed light in to this area as these presentations may be seen in tropics more frequently.

\section{Conclusions}

Chest wall tuberculous cold abscess is a rare form of extrapulmonary TB. Prompt suspicion in a tropical setting with excision when it is large or when there is no significant improvement with oral anti-TB medication, may hasten recovery. Obtaining histological specimens to detect TB would help to confirm and refute other diagnoses in tropical setting where other pyogenic abscesses are frequently observed.

\section{References}

1. Faure E, Souilamas R, Riquet M, Chehab A, Le PimpecBarthes F, Manac'h D, Debesse B. Cold abscess of the chest wall: a surgical entity?. The Annals of Thoracic Surgery 1998 Oct 1; 66(4): 1174-8.

2. Kim YJ, Jeon HJ, Kim CH, Park JY, Jung TH, Lee EB, Park TI, Jeon KN, Jung CY, Cha SI. Chest wall tuberculosis: clinical features and treatment outcomes. Tuberculosis and Respiratory Diseases 2009 Oct 1; 67(4): 318-24.

3. Papavramidis TS, Papadopoulos VN, Michalopoulos A, Paramythiotis D, Potsi S, Raptou G, Kalogera-Foutzila A, Harlaftis N. Anterior chest wall tuberculous abscess: a case report. Journal of Medical Case Reports. 2007 Dec; 1(1): 152 .

4. Aghajanzadeh M, Pourrasouli Z, Aghajanzadeh G, MASAHNIA S. Surgical treatment of chest wall tuberculosis.

5. Kuzucu A, Soysal Ö, Günen H. The role of surgery in chest wall tuberculosis. Interactive Cardiovascular and Thoracic Surgery, 2004 Mar 1;3(1): 99-103. 
6. Morris BS, Maheshwari M, Chalwa A. Chest wall tuberculosis: a review of $\mathrm{CT}$ appearances. The British Journal of Radiology, 2004 May; 77(917): 449-57.

7. Gharwade CC, Jawade PG, Banerjee S, Bindu A, Gharwade SC. Forearm intramuscular tuberculosis of flexor digitorum profundus: a case report. International Surgery Journal, 2016 Dec 14; 2(4): 741-5.

8. Dabó H, Mineiro A, Carmelino J, Carvalho A, Gomes C. Skeletal muscle tuberculosis in an immunocompetent patient. Archivos de Bronconeumologia (English Edition). 2016; 6(52):340-1.

9. Elshafie KT, Al-Hinai MM, Al-Habsi HA, Al-Hattali MS, Hassan O, Al-Sukaiti R. A massive tuberculosis abscess at the erector spinae muscles and subcutaneous tissues in a young man. Sultan Qaboos University Medical Journal, 2013 Nov; 13(4): 601.

10. Lai KL, Shi ZY, Chao WC, Liu PY, Wu LH. Tuberculous muscle abscess. QJM: An International Journal of Medicine, 2014 Jan 20; 107(8): 683-4.
11. Do Cho K, Cho DG, Jo MS, ImAhn M, Park CB. Current surgical therapy for patients with tuberculous abscess of the chest wall. The Annals of Thoracic Surgery, 2006 Apr 1; 81(4): 1220-6.

12. Kamiyoshihara M, Ibe T, Iwasaki Y, Takise A, Itou H, Takeyoshi I. Chest wall tuberculoma with tumor-like appearance. Respiratory Medicine CME, 2010 Jan 1; 3(2): 98-100.

13. Chen CH, Shih JF, Wang LS, Perng RP. Tuberculous subcutaneous abscess: an analysis of seven cases. Tubercle and Lung Disease, 1996 Apr 1; 77(2): 184-7.

14. Hsu HS, Wang LS, Wu YC, Fahn HJ, Huang MH. Management of primary chest wall tuberculosis. Scandinavian Journal of Thoracic and Cardiovascular Surgery, 1995 Jan 1;29(3): 119-23. 